\title{
CURRENT TRENDS IN THE VISUAL TRANSFORMATION OF PERIODIC PUBLICATIONS IN THE FIELD OF MEDICINE AND SOCIAL CARE
}

\author{
Venelin Terziev ${ }^{1}$ and Silva Vasileva ${ }^{2}$ \\ ${ }^{1}$ Full Member of the Russian Academy of Natural History, Professor, Eng., D.Sc. (National \\ Security), D.Sc. (Economics), D.Sc. (Social Activities), Ph.D. Georgi Rakovski Military Academy, \\ Sofia, Bulgaria; Kaneff University Hospital, Rousse, Bulgaria, vkterziev@gmail.com \\ ${ }^{2}$ Yordan Yovkov High School, Rousse, Bulgaria, silvastvasileva@gmail.com
}

\begin{abstract}
Contemporary trends in book publishing, "supported" by the imposition of online communication by means of information technologies, necessitated a complete change in the vision and presentation of the media in general, and for specialized periodicals in particular. The destiny of specialized publications follows the line of visual transformation of other publications. Although slower, this process is also observed in Bulgarian periodicals, incl. journals in the field of medicine and social care. The transition has been smooth, initially involving a long co-existence of both print and online editions, leading to a complete transition of e-editions.
\end{abstract}

Keywords: visual transformation, periodic publications, medicine and social care.

\section{INTRODUCTION}

The global pandemic situation has definitely raised the issue of health awareness of the population as a major priority in our daily lives. Currently, special attention is paid to the question of the reliability of this information. Consequently, it is extremely important for the right people and teams to be in the right place, to cooperate and provide high quality and effective care for their patients - all experts at the forefront of health care are considered (Alexander, 2021a). It is also essential that they use the right knowledge and evidence at the right time. This is to be achieved through timely sharing of knowledge, experience and practice, which, in turn, leads to improved results in the mission of each person, regardless of their professional field or scope of interests. Here, too, the role of medical periodicals is coming to the fore as a means for sharing knowledge and a window for accessing latest and most up-to-date information. A role that has a long history, and its brief overview back in time will make it easier and possible to rediscover the potential of this type of publications to provide health knowledge today.

In his book Mediamorphosis: Understanding New Media, Roger Fiedler discusses three major periods in human communication that play a central role in the progress and expansion of civilization - oral, written, and digital (Fiedler, 2005). After the age of the spoken word as the only culture and knowledge bearer, the occurrence of the written word changed the course of history and caused the second media morphosis. Writing allows information and knowledge to be stored and passed on to generations, so it is not surprising that it was after this media morphosis that the first ancient civilizations and empires emerged (Bonev, 2021b). Facing the third major media morphosis - namely digitalization, the process of digitalization of the 
media has begun, including printed editions. Threatened with extinction, as their very medium of information - paper - is being replaced by digital medium, they are increasingly being distributed online. The new media, to which the printed periodicals quickly turned, have a completely different language and require their authors to communicate with the users through its means of communication and expression (Bonev, 2021b).

\section{CURRENT TRENDS IN THE VISUAL TRANSFORMATION OF PERIODIC PUBLICATIONS IN THE FIELD OF MEDICINE AND SOCIAL CARE}

Contemporary trends in book publishing, "supported" by the imposition of online communication by means of information technologies, necessitated a complete change in the vision and presentation of the media in general, and for specialized periodicals in particular. The destiny of specialized publications follows the line of visual transformation of other publications. Although slower, this process is also observed in Bulgarian periodicals, incl. journals in the field of medicine and social care. The transition has been smooth, initially involving a long co-existence of both print and online editions, leading to a complete transition of e-editions.

\subsection{Publications in Traditional Format}

According to the electronic catalogue of the National Library "St. St. Cyril and Methodius" from 2000 to May 2021, 82 medical journals have been published in Bulgaria, 11 of which ceased being published. Out of all 82 titles, only one - Prevention and Rehabilitation, is issued on a portable electronic medium CDR. For the rest of the titles, it is noticeable that most of them continue to be published only on paper. Nineteen publications have their own website or other platform - most often of the institution or organization they belong to, where only primary bibliographic information is published: editorial team, reviewers, authors, leading titles, etc. Such are the journals Modern Medicine, Health and Science and others.

Contemporary Medicine journal was established in 1950 as a successor to the journal Medical Chronicles, which had inherited in 1948 the periodical of the then Medical Union. It is a multidisciplinary journal with a scientific and educational character, with the longest history in the medical periodical press. Today, the journal is a fact thanks to the ambition of the Union of Medical Specialists (SBMS). Contemporary Medicine concept is based on the real need for modern knowledge in the field of medicine and the need for its practical application in term of health promotion, prevention, medical ethics and health care. The editorial board includes prominent leading Bulgarian specialists and academic staff working in various medical fields. The editor-in-chief is Prof. Dr. Boris Bogov - Executive Director of Alexandrovska University Hospital. The prominent specialist was a national consultant in nephrology in the period 2016 - 2018, and since 2018 he has been chairman of the Expert Council on Nephrology under the Ministry of Health. Since 2016 he has been Chairman of the Standing Committee for Health and Sports at the National Agency for Evaluation and Accreditation.

The journal covers almost all branches of medicine - from "exotic" aviation, marine and space medicine to the well-known radiology and rheumatology. Information about the journal can be found on the website of the Union of Bulgarian Medical Specialists and the Union of Bulgarian Medical Societies. A future goal of this magazine is to get published in a digital format and thus reaching the target audience more effectively and increasing the specific qualification in the field of medicine and public health of a wider range of health professionals. There is no electronic archive of the publication.

Health and Science magazine began to get published in 2011 by Yordanka Filaretova Medical College at the Medical University, Sofia. The Publishing Board and the Editorial Board include specialists from various fields of preclinical and clinical medicine, health promotion and prevention, health care, medical ethics, public health, health management and administration. The publication is multi-profile, and the purpose of its publishers is to be a source of professional information for education, science-based prevention, treatment and rehabilitation of diseases and research in the field of medical sciences and health system.

The publication prints interesting clinical cases, modern knowledge, current scientific projects and discused issues and opinions in the field of medical sciences. In the regular rubrics of the magazine, readers can find clinical reports, scientific articles, research, analysis, reviews, regulations, current information about published textbooks, manuals and more.

The magazine is published only in traditional format.

\subsection{Mixed Type Editions}

Most of the editions are of mixed type - published in traditional and digital format. Here we will focus on two of them, and through them we'll try to represent the most typical ones of the mixed type.

Medical Meridians Magazine: Magazine for Strategic Health Management was founded in 2010 by Prof. 
Veselin Borisov, MD. and Assoc. Prof. Petar Gornenski, Ph.D. It is a publication of Balkan History and Philosophy of Medicine Association and Sustainable Development Center. The journal 's editor-in-chief is Acad. Prof. Veselin Borisov - Doctor of Medical Sciences, Professor of Social Medicine, Health Policy and Management at Sofia Medical University - Faculty of Public Health. Medical Meridians is published with the expert partnership of the Faculty of Public Health - Sofia. Each issue has a separate topic, usually authored by a representative of the editorial team, and features 8 to 10 original articles.

The journal is referenced in Advances in Bulgarian Science and is registered in the National Center for Information and Documentation (NACID).

Military Medicine Journal is the official publication of the Military Medical Academy and the Bulgarian Scientific Society of Military Medicine. It has been published since 2003. Its first editor-in-chief is Brigadier General Associate Professor Stoyan Tonev, and since the third issue of 2017 the editor-in-chief has been Major General Prof. Dr. Ventsislav Mutafchiiski, MD, FACS. General Ventsislav Mutafchiiski is also the Head of the National Operational Headquarters for Fight with Coronavirus Pandemic in Bulgaria.

The narrow professional orientation has also determined the ever-present sections in the magazine. Rubrics such as "Reviews", "Cases from Practice", "Current Diagnostic and Treatment Problems" can be found from the first to the latest issue of the magazine.

It is characteristic of both editions - Medical Meridians: Journal of Strategic Health Management and Military Medicine that the digital versions are completely identical to the printed ones. Maintaining a digital archive practically duplicates paper. The issues lack interactive links from the content, as well as internal links. However, digital versions make it easier for readers to use them and search data and also help promote authors and their publications.

\subsection{Editions in Digital Format}

Economic prerequisites are one of the main reasons for the transition from traditional to digital versions of publications. But there are a number of other reasons for the widespread popularity of online publications among consumers:

- Interactivity;

- Possibility for personalized content;

- 24-hour publication of up-to-date information;

- Possibility to update the published materials;

- Access to the entire archive of the publication;

- Easier access to required information;

- Multimedia content;

- Notifications on user-defined topics. (Bonev, 2021b)

These advantages are forcing more and more publishers to abandon print media in favour of online versions. Online publishing is supporting another positive trend in the development and validation of specialized journals, namely their inclusion in reference bibliographic or full-text databases, which in turn increases the visibility of authors and publications. It is an interesting fact that despite their inclusion in global information resources, online publications remain outside the catalogue of the national bibliographic agency.

Of the specialized publications in the field of medicine, magazines in digital format only are not yet a mass phenomenon. Despite the mandatory legalization registration of each publication, the ISSN system still lacks statistics on publications.

Occupational Health and Safety Magazine is a publication of the National Centre for Public Health and Analysis of the Centre for International Information on Occupational Safety and Health of the International Labour Organization (ILO CIS Centre). This is a highly specialized scientifically applied publication in the field of health and safety at work and aims to popularize and promote research on risk factors for health and safety at work, assessment and control of health risks, problems of occupational health services; good practices and policies for the prevention of accidents at work, occupational and work-related diseases, promotion of health and workability. The journal publishes reviews, scientific articles, good practices, methodological materials, opinions, announcements of events, new books and more.

The editor is Assoc. Prof. Katya Vangelova, Ph.D. - Head of the Occupational Health Department at the National Centre for Public Health. The institutional nature of the publication determines its authors and 
affiliates - these are mainly employees of the centre, and the topics cover issues related to night work, working capability and the factors that affect it, assessment of working capability and more.

It has been published since 2015 in Bulgarian and only in digital format.

Bulgarian Journal of Public Health is an official publication of the National Centre for Public Health and Analysis. The publication is bilingual, multidisciplinary and includes publications in the field of health policy and practice, health management and economics, epidemiology of non-communicable and communicable diseases, public health, health promotion and disease prevention, environment and health, occupational medicine, food and nutrition, crisis situations and public health, mental health. The magazine provides a forum for discussion on current issues of public health in Bulgaria, Europe, USA and other countries. Materials on current topics, studies, synopses and reports from international and national scientific forums and round tables are also published in special appendices. The journal aims to popularize and promote research, good practices, policies, governance and public health education.

Bulgarian Magazine for Public Health has been published since 2009. Its editor-in-chief is Prof. Dr. Petko Salchev, MD. The International Advisory Board includes scientists from the United States, the United Kingdom, Portugal, Macedonia and others. The magazine is published in 4 books a year and is published only in digital format.

Both magazines - Occupational Health and Safety Magazine and Bulgarian Magazine for Public Health - are publications of the same organization and have a similar visual layout. Both editions lack in-text links and hyperlinks.

The publication of two new electronic magazines in the field of healthcare has recently started in Bulgaria. The choice of titles is determined by the similar innovative approach in the choice of format by the teams and at the same time - the different fate of the two editions.

Bulgarian Science and Medicine Magazine is a project of a team that has been disseminating scientific and popular science information for 11 years, publishing Nauka Magazine. In 2016, an online publication dedicated entirely to medicine was launched, namely the magazine Bulgarian Science and Medicine. The journal is an extension of their work as a scientific information portal. The e-version of the publication provides an opportunity for all interested readers to use the reading materials after making a paid registration. The editor-in-chief of the magazine is Petar Teodosiev, who is also the editor-in-chief of Bulgarian Science and Nauka.bg. In the introductory article of the magazine's first issue, the editor-in-chief defines the profile of the publication as "... suitable not only for specialists but also for anyone interested in modern medicine, regardless of age, profession and education." Aimed at the younger audience interested in the achievements of modern medicine, the journal is richly illustrated, and the format uses the advantages of modern technology - the publications are online and can be read after paid registration. As a part of its innovative practice, aimed at expanding access to materials for the visually impaired, issue \# 9 of the publication is the first audio number available in MP3 format

Despite the innovative approach and ambition of the young team, the magazine stopped being published in 2018.

In 2020, the first issue of the Scientific electronic journal Society and Health was published. The magazine is institutionalized at the Kaneff University Hospital in the city of Ruse (Bulgaria). The editor-in-chief of the magazine is Professor, D.Sc. (National Security), D.Sc. (Economics), D.Sc. (Social Activities), Eng.Venelin Terziev, and the editorial team includes representatives of 23 research institutes and universities from 8 countries.

The publication is a scientific platform for sharing knowledge, achievements and experience of researchers from different countries and fields of knowledge and provides an opportunity for doctors, researchers, PhD students, postgraduates and habilitated specialists from the University Hospital and other institutions and countries as well as areas of knowledge, to have a best opportunity for a quick access to publish their research and studies. The 4 issues of the magazine published so far include articles by more than 100 authors from over 10 countries.

The interdisciplinary nature of the publication allows it to publish articles related to social, social, economic sciences, administration and management, social activities, pedagogy and medicine. This is an opportunity for many scholars and researchers to be able to submit their drafts of interdisciplinary character or covering strictly profiled subject.

The initial decision, taken by the editorial team, to publish the magazine only online determines the visual layout of the publication. Publishing it through an online publishing platform provides this opportunity for an 
interactive vision and functionality. The in-text hyperlinks available facilitate movement in the publication corpus.

The online edition provides free access to scientific information, which is part of the trend for open science. In this context of open access, Society and Health Magazine is available on the internet platforms of the publishers and is included in the reference list of the Bulgarian Ministry of Education and Science. It is distributed through the Research Gate (RG) platform, Social Science Research Network (SSRN) and is available in the Google Science Information System. It is also registered in the National Centre for Information and Documentation (NACID).

There are different predictions about when periodicals will completely cease to be printed on paper, switching to completely digital format. Francis Gurry, director general of the World Intellectual Property Organization (WIPO), affirms that this will happen worldwide in 2040 (Trincia, 2011). Only time will tell whether this prediction will come true. It is fact, however, that today editions of periodicals create multimedia content and in addition to written form, many of the articles are uploaded online as podcasts with audio files, and some of them even with video. Today's publishers need to be able to work with these formats, as well as with different web content management systems, engaged in various social media and networks in order to maintain a constant dialogue with their readers and to promote their articles.

\section{CONCLUSION}

Public health, which synthesizes the historical and, sometimes, political reality, can give a clear picture of the principles of governance, organization and trends in the development of both the health system and the state itself, while coverage of health issues truly represents the social and political development of a country. Specialized medical publications are an integral part of the process of disseminating health knowledge and establishing of health culture both among professionals and local community (Terziev, Vasileva, 2021c). Their pages reflect opinions, assessments, analyses and decisions taken by professional medical organizations or individual physicians on various important public issues, acts, administrative problems, etc., which are directly related to the medical community. A retrospective review of periodicals helps us see how this was happening in the past, and a comparison with contemporary practices reveals how these principles are applied today, allowing comparability with current trends and practices.

\section{REFERENCE LIST}

Alexander, R. (2021a). 5 Reasons Knowledge Management Is Crucial in the Healthcare Industry. Excerpted from Bloomfire:

https://bloomfire.com/blog/3-reasons-knowledge-management-critical-healthcareindustry/\#: :text=It\%E2\%80\%99s\%20clear\%20that\%20healthcare\%20is\%20a\%20knowledgedriven\%20industry.,can\%20ultimately\%20lead\%20to\%20increased\%20efficiency\%20and\%20producti vity, 01/2021.

Fiedler, R. (2005). Mediamorphosis: Understanding New Media. Sofia: Kralica Mab Publishing.

Bonev, H. (2021b). The print media in the digital age. Excerpted from

http://ebox.nbu.bg/: http://ebox.nbu.bg/mascom12/view_lesson.php?id=19, 01/2021.

Trincia, C. (2011). WIPO DG Francis Gurry: Newspapers Going Extinct. Excerpted from The Global Journal: https://theglobaljournal.net/article/view/254/, 09/2021.

Terziev, V., Vasileva, S. (2021c). The public library as health information resource, 2021. 